\title{
Nonlocal quantum effective actions in Weyl-Flat spacetimes
}

\author{
Teresa Bautista, ${ }^{a}$ André Benevides ${ }^{b, c}$ and Atish Dabholkar ${ }^{c, d, e}$ \\ ${ }^{a}$ Max Planck Institute for Gravitational Physics (Albert Einstein Institute), \\ Mühlenberg 1, D-14476 Potsdam, Germany \\ ${ }^{b}$ Scuola Internazionale Superiore di Studi Avanzati (SISSA), \\ Via Bonomea 265, Trieste 34136 Italy \\ ${ }^{c}$ Abdus Salam International Centre for Theoretical Physics, \\ Strada Costiera 11, Trieste 34151 Italy \\ 'Sorbonne Universités, UPMC Univ Paris 06, UMR 7589, LPTHE, \\ Paris, F-75005 France \\ ${ }^{e}$ CNRS, UMR 7589, LPTHE, \\ Paris, F-75005 France \\ E-mail: teresa.bautista@aei.mpg.de, afeitosa@sissa.it, atish@ictp.it
}

ABSTRACT: Virtual massless particles in quantum loops lead to nonlocal effects which can have interesting consequences, for example, for primordial magnetogenesis in cosmology or for computing finite $N$ corrections in holography. We describe how the quantum effective actions summarizing these effects can be computed efficiently for Weyl-flat metrics by integrating the Weyl anomaly or, equivalently, the local renormalization group equation. This method relies only on the local Schwinger-DeWitt expansion of the heat kernel and allows for a re-summation of the anomalous leading large logarithms of the scale factor, $\log a(x)$, in situations where the Weyl factor changes by several e-foldings. As an illustration, we obtain the quantum effective action for the Yang-Mills field coupled to massless matter, and the self-interacting massless scalar field. Our action reduces to the nonlocal action obtained using the Barvinsky-Vilkovisky covariant perturbation theory in the regime $R^{2} \ll \nabla^{2} R$ for a typical curvature scale $R$, but has a greater range of validity effectively re-summing the covariant perturbation theory to all orders in curvatures. In particular, it is applicable also in the opposite regime $R^{2} \gg \nabla^{2} R$, which is often of interest in cosmology.

Keywords: Anomalies in Field and String Theories, Effective Field Theories, Renormalization Group, Models of Quantum Gravity

ArXiv ePrint: 1711.00135 


\section{Contents}

1 Introduction 1

2 Effective actions from Weyl anomalies 3

2.1 Classical actions and the background field method 4

$\begin{array}{lll}2.2 & \text { Weyl anomaly and the local renormalization group }\end{array}$

$\begin{array}{llr}2.3 & \text { Integration of the Weyl anomaly } & 9\end{array}$

$\begin{array}{ll}2.4 & \text { Schwinger-DeWitt expansion of the heat kernel } \\ \end{array}$

3 Nonlocal effective actions $\quad 12$

$\begin{array}{lll}3.1 & \text { The Polyakov action in two dimensions } & 13\end{array}$

$\begin{array}{ll}3.2 & \text { Quantum effective action for Yang-Mills theory } \\ \end{array}$

$\begin{array}{ll}3.3 & \text { Quantum effective action for a self-interacting scalar field }\end{array}$

$\begin{array}{ll}3.4 & \text { Equations of motion } \\ 3.5 & 16\end{array}$

$\begin{array}{ll}\text { 3.5 Barvinsky-Vilkovisky expansion and conformal decomposition } & 17\end{array}$

\section{Introduction}

Virtual massless particles in quantum loops lead to nonlocal effects. The quantum dynamics of such massless particles coupled to a slowly evolving metric is summarized by the one-particle-irreducible (1PI) quantum effective action for the background fields obtained by integrating out the quantum loops. Unlike the Wilsonian effective action, the 1PI effective action necessarily contains nonlocal terms which are not derivatively suppressed. These nonlocal terms can have interesting consequences, for example, for primordial magnetogenesis in cosmology or for computing finite $N$ corrections in AdS/CFT holography.

The computation of the nonlocal quantum effective action is in principle a well-posed problem in perturbation theory. One can regularize the path integral covariantly using dimensional regularization or short proper-time regularization and evaluate the effective action using the background field method. However, explicit evaluation of the path integral is forbiddingly difficult. For instance, to obtain the one-loop effective action it is necessary to compute the heat kernel of a Laplace-like operator in an arbitrary background, which amounts to solving the Schrödinger problem for an arbitrary potential. For short proper time, the heat kernel can be computed using the Schwinger-DeWitt expansion $[1,2]$ which is analogous to the high temperature expansion. This is adequate for renormalizing the local ultraviolet divergences and to obtain the Wilsonian effective action if the proper time is short compared to the typical radius of curvature or the Compton wavelength of the particle being integrated out. However, the nonlocal 1PI effective action receives contributions from the entire range of the proper time integral and the Schwinger-DeWitt expansion is in general not adequate. 
To obtain the full nonlocal effective action, one could use the covariant nonlocal expansion of the heat kernel developed by Barvinsky, Vilkovisky, and collaborators [3, 4]. The effective action in this expansion has been worked out to third order in curvatures in a series of important papers [5-8] and illuminates a number of subtle issues, for example, concerning anomalies and the Riegert action [9-13]. However, for a general metric the explicit expressions are rather complicated already at the third order. Furthermore, the Barvinsky-Vilkovisky (BV) expansion requires $\mathcal{R}^{2} \ll \nabla^{2} \mathcal{R}$, where $\mathcal{R}$ denotes a generalized curvature including both a typical geometric curvature $R$ as well as a typical gauge field strength $F$. One is often interested though in the regime of slowly varying curvatures, $R^{2} \gg \nabla^{2} R$, for example during slow-roll inflation. This is beyond the validity of the $\mathrm{BV}$ regime.

The aim of the present work is to find practical methods to go beyond these limitations but only for a restricted class of metrics that are Weyl-flat. In this case, one can exploit the symmetries of the problem. The only dynamical mode of the background metric is the Weyl factor which is a single function. For simplicity we further restrict ourselves to classically Weyl invariant actions, since these allow for a very straightforward computation of the Weyl anomaly. The latter is the Weyl variation of the action which can be viewed as a first order scalar functional equation for the action that can be easily integrated. The initial value of the action functional can often be determined by the flat space results. In this manner, the entire effective action including its anomalous dependence on the Weyl factor can be determined efficiently.

The main advantage of our approach is that one can extract the essential physics with relative ease. Weyl anomalous dimensions of local operators (or equivalently the beta functions) can be computed reliably using local computations such as the Schwinger-DeWitt expansion. The resulting actions are necessarily nonlocal much like the Wess-Zumino action for chiral anomalies. ${ }^{1}$ Even though we relax the restriction $R^{2} \ll \nabla^{2} R$, we still need to assume $F^{2} \ll \nabla^{2} F$ for a typical field strength $F$. In summary, the Barvinsky-Vilkovisky regime requires rapidly varying curvature as well as rapidly varying field-strength whereas our regime requires only rapidly varying field-strength. Our method essentially re-sums the BV expansion to all orders in geometric curvatures albeit for a restricted class of Weyl-flat metrics as we discuss in section 3.5.

These nonlocal actions for Weyl-flat metrics can have a number of interesting applications. In $A d S / C F T$ correspondence, Weyl-flat metrics are relevant for the bulk description of renormalization group flows in the boundary CFT. Loop effects of massless supergravity fields are important, for example, in the computation of finite $N$ effects in the bulk such as the finite charge corrections to the Bekenstein-Hawking entropy of black holes [14-17]. In cosmology, the Robertson-Walker metric for an isotropic and homogeneous universe with flat spatial section is Weyl flat. During many epochs in the early universe, various particles can be massless or nearly massless compared to the Hubble scale. Quantum loops of these particles can lead to an anomalous dependence on the Weyl factor which can have

\footnotetext{
${ }^{1}$ The chiral anomaly itself can be deduced from local Schwinger-DeWitt expansion. The nonlocal WessZumino action is then obtained by the Wess-Zumino construction which essentially integrates the local anomaly equation. Our method extends this procedure to situations with nontrivial beta functions.
} 
interesting consequences. For example, in massless electrodynamics it can contribute to the generation of primordial magnetic fields [18-23] where one is precisely in the regime of rapidly varying field strengths but slowly varying curvatures. This approach can also be useful for exploring the stability of de Sitter spacetime, and the cosmological evolution of the Weyl factor and other physical parameters in quasi de Sitter spacetimes in four dimensions similar to the two-dimensional models analyzed in [24-26]. Possible implications of nonlocal actions have been explored, for example, in [27-34].

Our method naturally lends itself to a re-summation of leading large logarithms using a 'local renormalization group improvement' as we discuss in section 3.3. These are anomalous logarithms of the scale factor of the metric, $\log a(x)$, where $g_{\mu \nu}=a^{2}(x) \eta_{\mu \nu}{ }^{2}$ In cosmology, one is dealing with extremely long times and distances spanning several logarithmic scales. The scale factor of the universe underwent at least 60 and possibly more $e$-foldings. Thus, large logarithms could render perturbation theory invalid. Framing the method of Weyl anomaly integration within the formalism of the local RG is thus useful to extend the effective action beyond the perturbative limit of small logarithms, by resumming the higher-loop powers of $\log a(x)$. These logarithmic quantum corrections add up to something appreciable and have a potential for interesting applications if they can be properly re-summed.

The paper is organized as follows. In section 2, we describe how the Weyl anomaly can be used to determine the quantum effective action with the help of an 'integration lemma' and the Schwinger-DeWitt expansion of the heat kernel. In section 3 we apply this method to compute the effective action for the Yang-Mills field coupled to massless matter and for a self-interacting massless scalar field. In section 3.5, we discuss the relation of our results to the nonlocal covariant perturbation theory developed by Barvinsky-Vilkovisky and similar results obtained by Donoghue and El-Menoufi [35, 36] using Feynman diagrams in the weak field limit.

\section{Effective actions from Weyl anomalies}

In this section we describe the general method for computing the quantum effective action at the one-loop order for essentially all the standard model fields in Weyl-flat spacetimes by integrating the Weyl anomaly. To simplify the discussion, we ignore Yukawa couplings and work in the conformal massless limit so that all couplings are dimensionless. Classical Weyl invariance is not fundamentally necessary to integrate the Weyl anomaly in order to compute the effective action. However, it does simplify the computation of the anomaly. Dimensionful couplings and non-conformal scalars can be incorporated with some modifications [37]. We first review elements of the background field method and gauge fixing to

\footnotetext{
${ }^{2}$ This re-summation of large logarithms should not be confused with the re-summation of the BV expansion mentioned two paragraphs above. The latter is an effective re-summation of the BV expansion to all orders in the geometric curvatures in the case of Weyl-flat backgrounds, thus reproducing the one-loop effective action that we obtain from Weyl anomaly integration. The one we mention here is a further re-summation of higher-loop powers of the conformal factor $\Omega(x)=\log a(x)$ or 'leading large logarithms' in the usual sense of the RG.
} 
set up our conventions. We then discuss the anomalies in terms of the Schwinger-DeWitt expansion and a lemma to obtain the effective action by integrating the anomaly.

\subsection{Classical actions and the background field method}

Consider the classical action for a conformally coupled real scalar field $\varphi$ with quartic self-interaction:

$$
\mathcal{I}_{0}[g, \varphi]=-\int d^{4} x \sqrt{|g|}\left[\frac{1}{2}|\nabla \varphi|^{2}+\frac{1}{12} R \varphi^{2}+\frac{\lambda_{0}}{4 !} \varphi^{4}\right],
$$

where $\lambda_{0}$ is the bare coupling and $R$ the Ricci scalar for the metric $g$. This can also be viewed as the bare action in the ultraviolet if we regard the fields as bare fields. Even though we are interested in the Lorentzian action, for subsequent computations it is convenient to use the Wick-rotated action on the Euclidean section:

$$
\mathcal{S}_{0}[g, \varphi]=\int d^{4} x \sqrt{|g|}\left[\frac{1}{2}|\nabla \varphi|^{2}+\frac{1}{12} R \varphi^{2}+\frac{\lambda_{0}}{4 !} \varphi^{4}\right] .
$$

We denote the Lorentzian action by $\mathcal{I}$ and the Euclidean action by $\mathcal{S}$. Wick rotation of Lorentzian time $t$ to Euclidean time $t_{E}$ can be thought of as a coordinate change $t=-i t_{E}$ in the complexified spacetime. Tensors transform as tensors under this coordinate change and in particular the Lagrangian transforms as a scalar. The path integral is defined with weight $e^{i \mathcal{I}_{0}}$ in Lorentzian spacetime but with $e^{-\mathcal{S}_{0}}$ in Euclidean space. Using the fact that the volume element $\sqrt{|g|}$ equals $\sqrt{-g}$ on Lorentzian section but $\sqrt{g}$ on the Euclidean section, the two actions are simply related by $\mathcal{I}[g, \varphi] \rightarrow-\mathcal{S}[g, \varphi]$ as above.

In the background field method [38], one splits the quantum field as $\hat{\varphi}=\varphi+Q$, a sum of a background field $\varphi$ and the quantum fluctuations $Q$ around this background. The quantum effective action $\mathcal{S}[\varphi]$ for the background field $\varphi$ is then given by the path integral

$$
\exp (-\mathcal{S}[g, \varphi]):=\int \mathcal{D} Q \exp \left(-\mathcal{S}_{0}[g, Q+\varphi]-J[\varphi] Q\right),
$$

where the external current

$$
J[\varphi](x)=\frac{\delta \mathcal{S}[\varphi]}{\delta \varphi(x)}=\frac{\delta \mathcal{S}_{0}[\varphi]}{\delta \varphi(x)}+\ldots
$$

is a function of the background field adjusted so that the tadpoles vanish order by order in perturbation theory. We use short-proper time cutoff as a manifestly covariant regulator in the heat kernel method as described below. In renormalized perturbation theory, the UV divergences are renormalized with appropriately chosen counter-terms and all physical quantities are expressed in terms of the renormalized coupling $\lambda$ defined at a mass scale $M$. At one-loop order, the path integral can be approximated by the Gaussian functional integral

$$
e^{-\mathcal{S}_{1}[g, \varphi]}=e^{-\mathcal{S}_{0}[g, \varphi]} \int \mathcal{D} Q e^{-\frac{1}{2}\left\langle Q\left|\mathcal{O}_{\varphi}\right| Q\right\rangle},
$$

where $\mathcal{O}_{\varphi}$ is the quadratic fluctuation operator in the background:

$$
\mathcal{O}_{\varphi}=-\nabla^{2}+\frac{\lambda \varphi^{2}}{2}+\frac{1}{6} R
$$


with

$$
\nabla^{2}=\frac{1}{\sqrt{|g|}} \partial_{\mu}\left(\sqrt{|g|} g^{\mu \nu} \partial_{\nu}\right)
$$

The Gaussian integral can be evaluated in terms of the determinant of $\mathcal{O}_{\varphi}$,

$$
\mathcal{S}_{1}=\mathcal{S}_{0}+\frac{1}{2} \log \operatorname{det}\left(\mathcal{O}_{\varphi}\right)=\mathcal{S}_{0}+\frac{1}{2} \operatorname{Tr} \log \left(\mathcal{O}_{\varphi}\right)
$$

We use the convention

$$
\int d^{4} x \sqrt{|g|}|x\rangle\langle x|=\mathbf{1} ; \quad \operatorname{Tr}(\mathcal{O})=\int d^{4} x \sqrt{|g|}\langle x|\operatorname{tr} \mathcal{O}| x\rangle .
$$

We next consider gauge theory, concretely an $\mathrm{SU}(N)$ Yang-Mills field coupled to a massless complex scalar and a massless Dirac fermion transforming in the fundamental representation. The classical Lorentzian action is

$$
\mathcal{I}_{0}[g, A]=-\int d^{4} x \sqrt{|g|}\left[\frac{1}{4 e_{0}^{2}} F_{\mu \nu}^{a} F^{a \mu \nu}+|D \Phi|^{2}+\frac{1}{6} R|\Phi|^{2}+i \bar{\Psi} \Gamma^{\alpha} e_{\alpha}^{\mu} D_{\mu} \Psi\right],
$$

where $e_{0}^{2}$ is the bare gauge coupling and $a$ is the adjoint index $\left(a=1,2, \ldots, N^{2}-1\right)$. The covariant derivative is now defined including both the spin and the gauge connection:

$$
D_{\mu}:=\partial_{\mu}+\frac{1}{2} w_{\mu}^{\alpha \beta} J_{\alpha \beta}+A_{\mu}^{a} T_{a}, \quad(\alpha, \beta=0, \ldots, 3),
$$

where $\left\{J_{\alpha \beta}\right\}$ are the Lorentz representation matrices and $\left\{T_{a}\right\}$ are the anti-Hermitian $\mathrm{SU}(N)$ representation matrices normalized so that $\operatorname{tr}_{F}\left(T_{a} T_{b}\right)=-\frac{1}{2} \delta_{a b}$ in the fundamental representation $F$. The quantum field $\hat{A}_{\mu}$ is a sum of a background $A_{\mu}$ and a quantum fluctuation $a_{\mu}, \hat{A_{\mu}}=A_{\mu}+a_{\mu}$. To choose the background gauge, the gauge transformation of the quantum gauge field

$$
\delta_{\epsilon} \hat{A}_{\mu}:=\hat{D}_{\mu} \epsilon=\partial_{\mu} \epsilon+\left[\hat{A}_{\mu}, \epsilon\right]
$$

can be split as

$$
\delta_{\epsilon} A_{\mu}=\partial_{\mu} \epsilon+\left[A_{\mu}, \epsilon\right]:=D_{\mu} \epsilon, \quad \delta_{\epsilon} a_{\mu}=\left[a_{\mu}, \epsilon\right] .
$$

It is convenient to choose the background gauge $D_{\mu} a^{\mu}=0$ so that the effective action for the background field is manifestly gauge invariant. We set the background fields for $\Phi$ and $\Psi$ to zero. Following the standard Fadeev-Popov procedure we add the gauge fixing term and ghost Euclidean actions which at one-loop are of the form

$$
\mathcal{S}_{g f}=\frac{1}{2 e_{0}^{2} \xi} \int d^{4} x \sqrt{|g|}\left|D_{\mu} a^{\mu}\right|^{2}, \quad \mathcal{S}_{g h}=-\int d^{4} x \sqrt{|g|} \bar{c} D^{2} c
$$

where the covariant derivatives contain only the background connection. We henceforth use the 't Hooft-Feynman gauge $\xi=1$.

The one-loop quantum effective action is then given by

$$
\mathcal{S}_{1}=\mathcal{S}_{0}+\operatorname{Tr} \log \left(\mathcal{O}_{\Phi}\right)-\frac{1}{2} \operatorname{Tr} \log \left(\mathcal{O}_{\psi}\right)+\frac{1}{2} \operatorname{Tr} \log \left(\mathcal{O}_{A}\right)-\operatorname{Tr} \log \left(\mathcal{O}_{c}\right) .
$$


The operators involved are typically of the second-order Laplace-type

$$
\mathcal{O}_{f}=-g^{\mu \nu} D_{\mu} D_{\nu} \mathbf{1}+\mathbf{E},
$$

where $D_{\mu}$ is the covariant derivative defined above which depends on the representation of the field, $\mathbf{1}$ is the identity in the representation space of the field, and $\mathbf{E}$ is the 'endomorphism matrix' that depends on the background fields.

The regularized functional trace for various operators $\mathcal{O}_{f}$ can be expressed in terms of the diagonal elements of the corresponding heat kernels $K_{f}(s):=e^{-s \mathcal{O}_{f}}$ by the standard expression:

$$
\begin{aligned}
\operatorname{Tr} \log \left(\mathcal{O}_{f}\right)=-\int_{\epsilon}^{\infty} \frac{d s}{s} \operatorname{Tr} K_{f}(s) & =-\int_{\epsilon}^{\infty} \frac{d s}{s} \int d^{4} x \sqrt{|g|}\left\langle x\left|\operatorname{tr} K_{f}(s)\right| x\right\rangle \\
& =-\int_{\epsilon}^{\infty} \frac{d s}{s} \int d^{4} x \sqrt{|g|} \operatorname{tr} K_{f}(x, x ; s) .
\end{aligned}
$$

Here ' $\operatorname{Tr}$ ' is a total trace including the spacetime 'index' $x$ as in (2.9) as well as the matrix indices of the Lorentz and $\mathrm{SU}(N)$ representations, whereas 'tr' is a trace over only the matrix indices. ${ }^{3}$ The short proper time cut-off $\epsilon$ has mass dimension -2 and hence we can write $\epsilon=M_{0}^{-2}$ and regard $M_{0}$ as the UV mass cutoff.

In general, it is not possible to evaluate $K_{f}(x, x ; s)$ explicitly for all values of the proper time. However, exploiting Weyl anomalies and the symmetries of Weyl-flat backgrounds, it is possible to compute $\mathcal{S}$ avoiding the proper time integral altogether, as we discuss in the next two sections.

\subsection{Weyl anomaly and the local renormalization group}

Since regularization with a short proper time cutoff $\epsilon$ is manifestly covariant, we do not expect any anomalies in the diffeomorphism invariance. On the other hand, the cutoff scale $M_{0}$ introduces a mass scale and there is a potential for Weyl anomalies.

The local Weyl transformation of the spacetime metric $g_{\mu \nu}$ is defined by

$$
g_{\mu \nu} \rightarrow e^{2 \xi(x)} g_{\mu \nu}, \quad g^{\mu \nu} \rightarrow e^{-2 \xi(x)} g^{\mu \nu},
$$

or infinitesimally,

$$
g^{\mu \nu}(x) \rightarrow g^{\mu \nu}(x)-2 \xi(x) g^{\mu \nu}(x) .
$$

All other fields we denote collectively as $\left\{\chi_{f}\right\}$ which transform with Weyl weights $\left\{\Delta_{f}\right\}$

$$
\chi_{f}(x) \rightarrow e^{-\Delta_{f} \xi(x)} \chi_{f}(x) .
$$

In particular, in four dimensions, a conformally coupled scalar field has Weyl weight 1, a fermion field has weight $3 / 2$, a gauge field has weight 0 so that the kinetic terms are scale

\footnotetext{
${ }^{3}$ See for example $[39,40]$ for notational conventions.
} 
invariant. The local Weyl group $\mathcal{G}$ is an infinite dimensional abelian group with generators $\left\{J_{x}\right\}$ acting on the space of fields: ${ }^{4}$

$$
J_{x}:=-2 g^{\mu \nu}(x) \frac{\delta}{\delta g^{\mu \nu}(x)}-\Delta_{f} \chi_{f}(x) \frac{\delta}{\delta \chi_{f}(x)} .
$$

Treating the coordinate $x$ of the local scaling parameter $\xi(x)$ as a continuous index, we can write an element of this group as

$$
e^{\xi \cdot J}
$$

with the 'summation' convention

$$
\xi \cdot J:=\sum_{x} \xi_{x} J_{x}:=\int d^{4} x \xi(x) J_{x}
$$

A Weyl-flat metric can be written as

$$
g_{\mu \nu}=e^{2 \Omega} \eta_{\mu \nu}=e^{\Omega \cdot J}(\eta)
$$

and is on the Weyl-orbit of the flat Minkowski metric $\eta_{\mu \nu}$.

Weyl invariance of the classical action implies that

$$
J_{x}\left(\mathcal{S}_{0}\left[g, \chi_{f}\right]\right)=0 .
$$

The cutoff $\epsilon$ required for defining the quantum path integral breaks Weyl invariance. Consequently the 1PI quantum effective action $\mathcal{S}$ for the background fields is no longer Weyl invariant. The quantum violation of classical Weyl invariance can be expressed as an anomaly equation:

$$
J_{x}\left(\mathcal{S}\left[g, \chi_{f}\right]\right):=\left(-2 g^{\mu \nu} \frac{\delta}{\delta g^{\mu \nu}(x)}-\Delta_{f} \chi_{f} \frac{\delta}{\delta \chi_{f}(x)}\right)\left(\mathcal{S}\left[g, \chi_{f}\right]\right)=-\mathcal{A}(x) \sqrt{|g|}
$$

where $\mathcal{A}(x)$ is the Weyl anomaly scalar. ${ }^{5}$ Since the violation of the Weyl symmetry is a result of the short-distance regulator, one expects on general grounds that the anomaly $\mathcal{A}$ must be local even though the 1PI action is generically nonlocal. In particular, it must admit a local expansion in terms of the background fields. The locality of the anomaly is

\footnotetext{
${ }^{4}$ Dimensionful couplings could be treated as additional 'spurion' scalar fields with Weyl dimensions equal to their classical mass dimensions so that the classical action is rendered Weyl invariant. This more general situation will be discussed in [37]. In this case, the background fields $\left\{\chi_{f}\right\}$ will include also the spurion fields.

${ }^{5}$ In conformal field theory, Weyl anomaly is usually understood to mean only the 'conformal anomaly', which arises from the Weyl non-invariance of the measure. This anomaly, which we denote by $\mathcal{C}(x)$, appears at the conformal fixed point of the theory, when all beta functions vanish, and consists of purely gravitational terms. Hence it only shows up when the theory is coupled to a curved metric. Besides the conformal anomalies, a second type of 'beta-function anomalies' $\mathcal{B}(x)$ arise when interactions are present. Interactions perturb the theory away from the fixed point, and nontrivial beta functions generate a renormalization group flow. In this case, the Weyl anomaly includes the beta function anomalies in addition to the conformal anomaly: $\mathcal{A}(x)=\mathcal{B}(x)+\mathcal{C}(x)$. Therefore, as opposed to the conformal anomaly, beta function anomalies do show up in flat spacetime. This distinction should not be confused with the Type-A and Type- $B$ classification of anomalies [41].
} 
of crucial importance. At one-loop, one can prove it explicitly and obtain a formula for the anomaly in terms of the local Schwinger-DeWitt expansion.

We illustrate the general argument for the conformally coupled scalar field $\varphi$. The infinitesimal Weyl variation of the quadratic action for the quantum fluctuation vanishes:

$$
\delta_{\xi}\left\langle Q\left|\mathcal{O}_{\varphi}\right| Q\right\rangle=\delta_{\xi} \int d^{4} x \sqrt{|g|} Q(x) \mathcal{O}_{\varphi} Q(x)=0 .
$$

Using the Weyl transformations of $Q$ and the background metric $g_{\mu \nu}$ we conclude that

$$
\delta_{\xi} \mathcal{O}_{\varphi}=-2 \delta \xi(x) \mathcal{O}_{\varphi}
$$

up to boundary terms. The quadratic fluctuation operator $\mathcal{O}_{\varphi}$ is thus covariant under Weyl transformations with weight 2 . It then follows that

$$
\begin{aligned}
\delta_{\xi} \mathcal{S}_{1}[g, \varphi] & =-\frac{1}{2} \int_{\epsilon}^{\infty} \frac{d s}{s} \operatorname{Tr} \delta e^{-s \mathcal{O}_{\varphi}}=\frac{1}{2} \int_{\epsilon}^{\infty} d s \operatorname{Tr}\left(\delta \mathcal{O}_{\varphi}\right) e^{-s \mathcal{O}_{\varphi}} \\
& =-\int_{\epsilon}^{\infty} d s \int d^{4} x \sqrt{|g|} \delta \xi(x)\left\langle x\left|\operatorname{tr} \mathcal{O}_{\varphi} e^{-s \mathcal{O}_{\varphi}}\right| x\right\rangle \\
& =\int_{\epsilon}^{\infty} d s \int d^{4} x \sqrt{|g|} \delta \xi(x) \frac{d}{d s}\left\langle x\left|\operatorname{tr} e^{-s \mathcal{O}_{\varphi}}\right| x\right\rangle .
\end{aligned}
$$

Performing the $s$ integral we obtain ${ }^{6}$

$$
\frac{\delta \mathcal{S}_{1}[g, \varphi]}{\delta \xi(x)}=J_{x}\left(\mathcal{S}_{1}[g, \varphi]\right)=-\left\langle x\left|\operatorname{tr} e^{-\epsilon \mathcal{O}_{\varphi}}\right| x\right\rangle \sqrt{|g|}=-\operatorname{tr} K_{\varphi}(x, x ; \epsilon) \sqrt{|g|} .
$$

A similar reasoning can be used for fermions since the Dirac action is Weyl invariant in all dimensions. For gauge fields, there is an additional subtlety because the gauge fixed action and the ghost action are not separately Weyl invariant. However, one obtains an analogous expression for the combined system of gauge and ghost fields [42]. Both for fermions and the gauge-ghosts system, the quadratic operators have Weyl weight two. The action of the Weyl generator on the field space is thus given by

$$
J_{x}\left(\mathcal{S}_{1}\left[g, \chi_{f}\right]\right):=-\mathcal{A}(x) \sqrt{|g|}=-2 \sum_{f} n_{f} \operatorname{tr} K_{f}(x, x ; \epsilon) \sqrt{|g|}
$$

where $n_{f}$ is the coefficient of $\operatorname{Tr} \log \left(\mathcal{O}_{f}\right)$ in (2.15) consistent with our convention in (2.17). Thus, $n_{\Phi}=1, n_{\Psi}=-\frac{1}{2}, n_{c}=-1, n_{A}=n_{\varphi}=\frac{1}{2}$.

Equation (2.33) shows the anomaly is determined entirely by the short proper time behavior of the heat kernel. Since the proper time cutoff $\epsilon$ effectively provides a covariant short-distance cutoff in spacetime, the resulting anomaly $\mathcal{A}(x)$ is local as promised. Therefore, it must admit an expansion in terms of local fields $V_{i}(x)$ : for the beta function anomaly,

$$
\mathcal{B}(x)=\sum_{i} \beta_{i} V_{i}(x)
$$

\footnotetext{
${ }^{6}$ If the operator $\mathcal{O}_{\varphi}$ has no zero modes there is no contribution from the upper limit of the integral.
} 
the $\mathcal{C}(x)$ anomaly is purely gravitational and has a similar expansion in terms of the local functionals of the metric such as the Euler density [43-45].

The Weyl anomaly equation is closely related to the local renormalization group [46-48] and the coefficients $\beta_{i}$ can be simply related to the usual beta functions. We illustrate this connection for Yang-Mills theory. The Weyl variation of the action with respect to the Weyl factor $\Omega$ of the metric (2.24) is given by (2.55) at one loop:

$$
J_{x}\left(\mathcal{S}_{1}[g, A]\right)=\frac{\delta \mathcal{S}_{1}[g, A]}{\delta \Omega(x)}=-\mathcal{B}(x) \sqrt{|g|}=\frac{b}{4} F^{2}(x) \sqrt{|g|},
$$

where $b$ is given by (2.56) and we have ignored the purely gravitational $\mathcal{C}(x)$ anomaly. To relate it to the local renormalization group, we note that a Weyl scaling of the metric increases length scales or decreases mass scales. Hence we can regard $M(x):=M e^{\Omega(x)}$ to be the position-dependent local renormalization scale ${ }^{7} M(x)$. Therefore,

$$
M(x) \frac{\delta}{\delta M(x)}=\frac{\delta}{\delta \Omega(x)} .
$$

If the scale $M(x)$ is position dependent, then it is natural to regard all renormalized couplings to be also position-dependent expectation values of nondynamical 'spurion' fields. For example, regarding, $1 / e^{2}=\lambda_{e}(x)$ as position dependent, and using (2.36) and (2.35) we conclude that

$$
\left[M(x) \frac{\delta}{\delta M(x)}+\beta_{e} \frac{\delta}{\delta \lambda_{e}(x)}\right] \mathcal{S}_{1}=0
$$

with

$$
\beta_{e}:=M \frac{d \lambda_{e}}{d M}=M \frac{d e^{-2}}{d M}=-b .
$$

For constant $M(x)$, functional derivatives are replaced by ordinary derivatives and one recovers the usual position-independent 'global' homogeneous renormalization group equation.

More generally, the local renormalization group equation is best thought of as a Weyl anomaly equation (2.26) with a local expansion for the anomaly $\mathcal{A}$.

\subsection{Integration of the Weyl anomaly}

Our goal is to deduce the nonlocal quantum effective action $\mathcal{S}\left[g, \chi_{f}\right]$ by integrating the local Weyl anomaly. Towards this end, we consider the following trivial identity ${ }^{8}$

$$
e^{\xi \cdot J}=\mathbf{1}+\int_{0}^{1} d t e^{t \xi \cdot J} \xi \cdot J
$$

We wish to compute $\mathcal{S}\left[g, \chi_{f}\right]$ for $\left(g, \chi_{f}\right)$ on the Weyl-orbit of $\left(\bar{g}, \bar{\chi}_{f}\right)$ with Weyl factor $\Omega(x)$ :

$$
\left(g, \chi_{f}\right)=e^{\Omega \cdot J}\left(\bar{g}, \bar{\chi}_{f}\right) .
$$

\footnotetext{
${ }^{7}$ This is true as long as one is dealing with 'primary' fields such as $g_{\mu \nu}$ or $F_{\mu \nu}$ which transform covariantly under Weyl transformation. In general, 'secondary' fields such as $R_{\mu \nu}$ or $\nabla_{\mu} \varphi$ are also relevant, which contain derivatives of the primary fields. In this case, the Weyl transformations contain terms with derivatives of the Weyl factor $\Omega$ and the equality (2.36) holds only up to these derivatives [37, 46, 47, 49-52].

${ }^{8}$ We thank Adam Schwimmer for this formulation.
} 
Using the identity above we obtain

$$
\begin{aligned}
\mathcal{S}\left[g, \chi_{f}\right] \equiv e^{\Omega \cdot J}\left(\mathcal{S}\left[\bar{g}, \bar{\chi}_{f}\right]\right) & =\left(\mathbf{1}+\int_{0}^{1} d t e^{t \Omega \cdot J} \Omega \cdot J\right)\left(\mathcal{S}\left[\bar{g}, \bar{\chi}_{f}\right]\right) \\
& =\mathcal{S}\left[\bar{g}, \bar{\chi}_{f}\right]+\int_{0}^{1} d t e^{t \Omega \cdot J} \Omega \cdot J\left(\mathcal{S}\left[\bar{g}, \bar{\chi}_{f}\right]\right) \\
& =\mathcal{S}\left[\bar{g}, \bar{\chi}_{f}\right]-\int_{0}^{1} d t e^{t \Omega \cdot J}\left(\int d^{4} x \Omega(x) \sqrt{|\bar{g}|} \mathcal{A}\left[\bar{g}, \bar{\chi}_{f}\right](x)\right)
\end{aligned}
$$

where we have used (2.26) in the last line. Using (2.40) we then conclude ${ }^{9}$

$$
\mathcal{S}\left[g, \chi_{f}\right]=\mathcal{S}\left[\bar{g}, \bar{\chi}_{f}\right]+\mathcal{S}_{\mathcal{A}}\left[\bar{g}, \Omega, \bar{\chi}_{f}\right],
$$

where

$$
\mathcal{S}_{\mathcal{A}}\left[\bar{g}, \Omega, \bar{\chi}_{f}\right]:=-\int_{0}^{1} d t \int d^{4} x \sqrt{\left|\bar{g} e^{2 t \Omega(x)}\right|} \Omega(x) \mathcal{A}\left[\bar{g} e^{2 t \Omega}, \bar{\chi}_{f} e^{-\Delta_{f} t \Omega}\right](x)
$$

is the contribution to the action from the anomaly. Lorentzian continuation of (2.44) gives a similar equation

$$
\mathcal{I}\left[g, \chi_{f}\right]=\mathcal{I}\left[\bar{g}, \bar{\chi}_{f}\right]+\mathcal{I}_{\mathcal{A}}\left[\bar{g}, \Omega, \bar{\chi}_{f}\right]
$$

but with $\mathcal{I}_{\mathcal{A}}$ given by

$$
\mathcal{I}_{\mathcal{A}}\left[\bar{g}, \Omega, \bar{\chi}_{f}\right]:=\int_{0}^{1} d t \int d^{4} x \sqrt{\left|\bar{g} e^{2 t \Omega(x)}\right|} \Omega(x) \mathcal{A}\left[\bar{g} e^{2 t \Omega}, \bar{\chi}_{f} e^{-\Delta_{f} t \Omega}\right](x)
$$

because the anomaly scalar does not change sign under Wick rotation.

Equation (2.44) is a simple identity that follows essentially from the group structure of Weyl transformations. It is thus applicable to any order in perturbation theory if we can compute the Weyl anomaly to that order. To compute the effective action to the oneloop order, one can use the expression (2.45) with the Weyl anomaly given in terms of the heat kernel as in (2.33). Since the short-time expansion of the heat kernel is determined by the local Schwinger-DeWitt expansion, we see that (2.44) enables us to determine the entire quantum effective action for Weyl-flat background metrics knowing only the local expansion.

Note that the left hand side of (2.46) depends only on the physical metric whereas the right hand side a priori depends on the fiducial metric $\bar{g}$ and $\Omega$ separately. It must therefore be true that the action on the right hand side exhibits 'fiducial Weyl gauge invariance'

$$
\bar{g} \rightarrow e^{2 \zeta(x)} \bar{g}, \quad \Omega \rightarrow \Omega-\zeta(x),
$$

under which the fiducial metric $\bar{g}$ transforms but the physical metric $g$ is invariant. This gauge invariance reflects the fact that splitting $g$ into $\bar{g}$ and $\Omega$ is ambiguous, and all splits related by a fiducial gauge transformation are physically equivalent. The fiducial gauge invariance of the right hand side of (2.46) is necessary to show that it depends only on the physical metric. As we explain in section 3.5, it is often far from obvious how the answer obtained using our method can be expressed covariantly entirely in terms of the physical metric. However, the procedure guarantees that this must be the case.

\footnotetext{
${ }^{9}$ The argument $g$ of the action $\mathcal{S}\left[g, \chi_{f}\right]$ functional here refers to the covariant tensor $g_{\mu \nu}$ and not $g^{\mu \nu}$.
} 


\subsection{Schwinger-DeWitt expansion of the heat kernel}

The trace of the heat kernel admits a short proper time expansion as

$$
\operatorname{Tr} K_{f}(\epsilon)=\int_{\mathcal{M}} d^{d} x \sqrt{|g|} \operatorname{tr} K_{f}(x, x ; \epsilon)=\int_{\mathcal{M}} d^{d} x \sqrt{|g|} \frac{1}{(4 \pi \epsilon)^{d / 2}} \sum_{n=0}^{\infty} a_{n}(x) \epsilon^{n} .
$$

The $a_{n}(x)$ are the Gilkey-Seeley-HaMiDeW [53-62] coefficients ${ }^{10}$ which are local scalar functions of the background fields. A general expression in any spacetime dimension is known explicitly for the first few of them in terms of $\mathbf{E}$ and geometric invariants.

Because the first $d / 2-1$ terms have negative powers of $\epsilon$, the above short time expansion is divergent. The divergences can be renormalized by appropriate local counterterms; the remaining finite piece is given by $a_{d / 2}(x)$ which thus determines the Weyl anomaly through (2.33).

The relevant $a_{n}(x)$ coefficients up to spacetime dimension $d=4$ are given by [40]

$$
\begin{aligned}
a_{0}= & \operatorname{tr} \mathbf{1} \\
a_{1}= & \operatorname{tr}\left(\frac{1}{6} R \mathbf{1}-\mathbf{E}\right) \\
a_{2}= & \operatorname{tr}\left(\frac{1}{2} \mathbf{E}^{2}-\frac{1}{6} \nabla^{2} \mathbf{E}-\frac{1}{6} R \mathbf{E}+\frac{1}{12} \Omega_{\mu \nu} \Omega^{\mu \nu}\right. \\
& \left.+\frac{1}{180}\left(6 \nabla^{2} R+\frac{5}{2} R^{2}-\frac{1}{2} E_{4}+\frac{3}{2} W^{2}\right) \mathbf{1}\right),
\end{aligned}
$$

where $\operatorname{tr} 1$ traces all indices, $\nabla_{\mu}:=\partial_{\mu}+\omega_{\mu}$ is the covariant derivative involving only the spin connection, and $\Omega_{\mu \nu}=\left[D_{\mu}, D_{\nu}\right]$ is the field strength of the full connection. $E_{4}$ is the Euler density in four dimensions and $W^{2}$ is the square of the Weyl tensor $W_{\mu \nu \rho}^{\sigma}$ defined by

$$
\begin{aligned}
E_{4} & =R_{\mu \nu \rho \sigma} R^{\mu \nu \rho \sigma}-4 R_{\mu \nu} R^{\mu \nu}+R^{2} \\
W^{2} & =R_{\mu \nu \rho \sigma} R^{\mu \nu \rho \sigma}-2 R_{\mu \nu} R^{\mu \nu}+\frac{1}{3} R^{2} .
\end{aligned}
$$

In four dimensions, the anomaly $\mathcal{A}=\mathcal{B}+\mathcal{C}$ is determined by $a_{2}(x)$. In table 1 we list the anomalies for the operators appearing in the Yang-Mills and conformally-coupled scalar actions. We have dropped the terms proportional to $\nabla^{2} R$ and $\nabla^{2} \varphi^{2}$. Such operators follow from the Weyl variation of local terms in the action, namely $R^{2}$ and $R \varphi^{2}$, hence are not genuine anomalies $[65,66]$. The vector potential operator $\mathcal{O}_{A}$ corresponds to the Feynman gauge $\xi=1$ and $F^{2}:=F_{\mu \nu}^{a} F^{a \mu \nu}$. Note that the $a_{2}(x)$ coefficients for the ghost and vector operators individually contain a term proportional to $R^{2}$. This is related to the the fact that the operators are not individually Weyl covariant. However, taken together, the $R^{2}$ terms cancel from the anomaly as expected from the Wess-Zumino consistency condition.

Putting these results together, the Weyl anomaly equation for Yang-Mills is

$$
J_{x}\left(\mathcal{S}_{1}[g, A]\right)=\frac{\delta \mathcal{S}_{1}[g, A]}{\delta \Omega}=\left(\frac{b}{4} F^{2}(x)-\mathcal{C}(x)\right) \sqrt{|g|},
$$

\footnotetext{
${ }^{10}$ After Hadamard, Minakshisundaram, and DeWitt [63, 64].
} 


\begin{tabular}{|c|c|c|c|}
\hline Field & $\mathcal{O}$ & $16 \pi^{2} \mathcal{B}$ & $16 \pi^{2} \mathcal{C}$ \\
\hline$\Phi$ & $-D^{2}+\frac{1}{6} R$ & $-\frac{1}{12} F^{2}$ & $\frac{N}{180}\left(-E_{4}+3 W^{2}\right)$ \\
$c, \bar{c}$ & $-D^{2}$ & $\frac{N}{6} F^{2}$ & $\frac{N^{2}-1}{180}\left(-5 R^{2}+E_{4}-3 W^{2}\right)$ \\
$A_{\mu}$ & $-D^{2} g^{\mu \nu}+R^{\mu \nu}-2 F^{\mu \nu}$ & $\frac{5 N}{3} F^{2}$ & $\frac{N^{2}-1}{180}\left(5 R^{2}-32 E_{4}+21 W^{2}\right)$ \\
$\Psi$ & $-D^{2}+\frac{1}{4} R-\frac{1}{2} F_{\mu \nu} \Gamma^{\mu} \Gamma^{\nu}$ & $-\frac{1}{3} F^{2}$ & $\frac{N}{180}\left(-\frac{11}{2} E_{4}+9 W^{2}\right)$ \\
$\varphi$ & $-\nabla^{2}+\frac{1}{6} R+\frac{1}{2} \lambda \varphi^{2}$ & $\frac{1}{8} \lambda^{2} \varphi^{4}$ & $\frac{1}{180}\left(-E_{4}+3 W^{2}\right)$ \\
\hline
\end{tabular}

Table 1. Weyl anomalies in $d=4$. The contributions from the complex scalars $\Phi$ and fermions $\Psi$ to the $\mathcal{B}$ anomaly are different for the abelian and non-abelian cases. In the table we have indicated the non-abelian ones relevant for Yang-Mills. For quantum electrodynamics, the contributions are multiplied by a factor of two due to the choice of normalization of the non-abelian gauge group generators.

with

$$
b=\frac{1}{48 \pi^{2}}\left(N_{S}+4 N_{F}-22 N\right)
$$

for an $\mathrm{SU}(N)$ theory with $N_{S}$ scalars and $N_{F}$ fermions in the fundamental. In quantum electrodynamics integrating out $N_{F}$ fermions and $N_{S}$ scalars, one would get a similar result with

$$
b=\frac{1}{24 \pi^{2}}\left(N_{S}+4 N_{F}\right) .
$$

For the real scalar field $\varphi$ with quartic self-interaction, we similarly obtain

$$
J_{x}\left(\mathcal{S}_{1}[g, \varphi]\right)=\frac{\delta \mathcal{S}_{1}[g, \varphi]}{\delta \Omega}=\left(-\frac{b \lambda}{4 !} \varphi^{4}(x)-\mathcal{C}(x)\right) \sqrt{|g|}
$$

with the beta function coefficient given by

$$
b=\frac{3 \lambda}{16 \pi^{2}} .
$$

\section{Nonlocal effective actions}

In this section we derive the one-loop quantum effective actions from the anomalies following the discussion in the previous section. We drop the subscript ' 1 ' used earlier to indicate the one-loop results. As a simple illustration, we first derive the two dimensional Polyakov action from the $\mathcal{C}(x)$ anomaly. In four dimensions, we ignore the $\mathcal{C}(x)$ anomaly and focus only on the $\mathcal{B}(x)$ anomaly to derive the effective action for the background fields $\Omega, A, \varphi$. 


\subsection{The Polyakov action in two dimensions}

The trace anomaly (2.26) for a massless free scalar in two dimensions is given by

$$
\mathcal{A}(x)=\operatorname{tr} K_{\varphi}(x, x, \epsilon) .
$$

The finite contribution to the trace in two dimensions is given by the coefficient $a_{1}(x)$ :

$$
\mathcal{A}(x)=\frac{1}{4 \pi} a_{1}(x)=\frac{1}{4 \pi} \operatorname{tr}\left(\frac{1}{6} R \mathbf{1}\right)=\frac{1}{24 \pi} R .
$$

In this case $\mathcal{B}=0$ and the anomaly is purely gravitational. Using (2.44) and the Weyl transformation for the Ricci scalar

$$
R=e^{-2 \Omega}\left(\bar{R}-2 \bar{\nabla}^{2} \Omega\right) \quad \text { for } \quad g=e^{2 \Omega} \bar{g},
$$

the effective action is given by $\mathcal{I}[g]=\mathcal{I}[\bar{g}]+\mathcal{I}_{\mathcal{C}}[\bar{g}, \Omega]$ with

$$
\begin{aligned}
\mathcal{I}_{\mathcal{C}}[\bar{g}, \Omega] & =\int_{0}^{1} d t \int d^{2} x \sqrt{\left|\bar{g} e^{2 t \Omega(x)}\right|} \Omega(x) \mathcal{A}\left[\bar{g} e^{2 t \Omega(x)}\right] \\
& =\frac{1}{24 \pi} \int_{0}^{1} d t \int d^{2} x \sqrt{|\bar{g}|} e^{2 t \Omega(x)} \Omega(x) e^{-2 t \Omega(x)}\left(\bar{R}-2 t \bar{\nabla}^{2} \Omega(x)\right) \\
& =\frac{1}{24 \pi} \int^{2} x \sqrt{|\bar{g}|}\left((\bar{\nabla} \Omega)^{2}+\bar{R} \Omega(x)\right),
\end{aligned}
$$

which is the Liouville action with the correct normalization. For $\bar{g}_{\mu \nu}=\delta_{\mu \nu}$, one can solve (3.3) for $\Omega$ in terms of $g$ using the fact that $\bar{R}=0$, and obtain the Polyakov action

$$
\mathcal{I}[g]=-\frac{1}{96 \pi} \int d^{2} x \sqrt{|g|} R \frac{1}{\nabla^{2}} R .
$$

Since in two dimensions every metric is Weyl flat, these results are valid for a general metric.

Under a fiducial Weyl transformation (2.48), the Liouville action is not invariant but transforms as

$$
\mathcal{I}_{\mathcal{C}}[\bar{g}, \Omega] \quad \rightarrow \quad \mathcal{I}_{\mathcal{C}}[\bar{g}, \Omega]-\frac{1}{24 \pi} \int d^{2} x \sqrt{|\bar{g}|}\left((\bar{\nabla} \zeta)^{2}+\bar{R} \zeta\right)
$$

However, the $\mathcal{I}[\bar{g}]$ also transforms as

$$
\mathcal{I}[\bar{g}]=-\frac{1}{96 \pi} \int d^{2} x \sqrt{|\bar{g}|} \bar{R} \frac{1}{\bar{\nabla}^{2}} \bar{R} \quad \rightarrow \quad \mathcal{I}[\bar{g}]+\frac{1}{24 \pi} \int d^{2} x \sqrt{|\bar{g}|}\left((\bar{\nabla} \zeta)^{2}+\bar{R} \zeta\right)
$$

ensuring the fiducial Weyl invariance of $\mathcal{I}[\bar{g}]+\mathcal{I}_{\mathcal{C}}[\bar{g}, \Omega]$. 


\subsection{Quantum effective action for Yang-Mills theory}

Applying (2.47) to the $\mathcal{B}$ anomaly of the Yang-Mills theory (2.55) in a Weyl-flat spacetime gives

$$
\mathcal{I}_{\mathcal{B}}[\eta, \Omega, A]=-\frac{b}{4} \int d^{4} x \eta^{\rho \alpha} \eta^{\sigma \beta} F_{\rho \sigma}^{a}(x) \Omega(x) F_{\alpha \beta}^{a}(x) .
$$

The flat space action can be easily determined from standard computations and is given by

$$
\mathcal{I}[\eta, A]=-\frac{1}{4 e^{2}(M)} \int d^{4} x d^{4} y \eta^{\rho \alpha} \eta^{\sigma \beta} F_{\rho \sigma}^{a}(x)\left\langle x\left|\left[1-\frac{b}{2} e^{2}(M) \log \left(\frac{-\partial^{2}}{M^{2}}\right)\right]\right| y\right\rangle F_{\alpha \beta}^{a}(y)
$$

where $-\partial^{2}$ is the flat-space d'Alembertian. The kets $|x\rangle$ here are normalized as in (2.9) but now with the flat metric $\eta$. The logarithm of an operator is defined by the spectral representation

$$
\log \left(\frac{\mathcal{O}}{M^{2}}\right)=\int_{0}^{\infty} d \mu^{2}\left(\frac{1}{M^{2}+\mu^{2}}-\frac{1}{\mathcal{O}+\mu^{2}}\right) .
$$

For the flat space d'Alembertian the logarithm can also be defined by a Fourier transform:

$$
\left\langle x\left|\log \left(\frac{-\partial^{2}}{M^{2}}\right)\right| y\right\rangle=\int \frac{d^{4} p}{(2 \pi)^{4}} e^{-i p \cdot(x-y)} \log \left(\frac{p^{2}}{M^{2}}\right) .
$$

Putting the two things together in (2.46) we conclude

$$
\mathcal{I}[g, A]=-\frac{1}{4 e^{2}(M)} \int d^{4} x \eta^{\rho \alpha} \eta^{\sigma \beta} F_{\rho \sigma}^{a}(x)\left[1-\frac{b}{2} e^{2}(M) \log \left(\frac{-\partial^{2}}{M^{2}}\right)+b e^{2}(M) \Omega(x)\right] F_{\alpha \beta}^{a}(x)
$$

where the logarithmic operator is to be understood as a bilocal expression integrated over $y$ as in (3.9). There is a gravitational piece coming from the $\mathcal{C}$ anomaly which we do not discuss.

Note that the action (3.8) arising from the anomaly follows from the local SchwingerDeWitt expansion and does not require any weak-field approximation. Thus, the main limitation in computing (3.12) comes from the evaluation of the flat space action (3.9). In (3.9) we have used the weak gauge field approximation $F^{4} \ll \nabla^{4} F^{2}$ as one normally does in flat space quantum field theory. It may be possible to compute the flat space action in other regimes, for example, in the regime of constant field strength. This can extend the range of validity of our results.

It is instructive to deduce this result using dimensional regularization. Again, the classical action (or the bare action in the UV) is given by

$$
\mathcal{I}_{0}[g, A]=-\frac{1}{4 e_{0}^{2}} \int d^{4} x \sqrt{|g|} g^{\rho \alpha} g^{\sigma \beta} F_{\rho \sigma}^{a} F_{\alpha \beta}^{a} .
$$

The classical energy momentum tensor

$$
T_{\mu \nu}^{c l}=\frac{1}{e_{0}^{2}}\left(F_{\mu \sigma}^{a} F_{\nu}^{a \sigma}-\frac{1}{4} g_{\mu \nu} F^{2}\right)
$$

is traceless. At the quantum level, the nonzero beta function implies a quantum violation of Weyl invariance. For a manifestly gauge-invariant computation of this Weyl anomaly 
we use dimensional regularization. In $4-\varepsilon$ dimensions, the bare coupling $e_{0}$ is related to the coupling $e$ renormalized at scale $M$ by

$$
\frac{1}{e_{0}^{2}}=M^{-\varepsilon}\left(\frac{1}{e^{2}}-\frac{b}{\varepsilon}\right), \quad M \frac{d e^{-2}}{d M}=-b .
$$

There is the usual pole coming from loop integrations of quantum fluctuations around a background field. For a Weyl-flat metric, the dimensionally regularized background field action depends only on the background gauge field and the Weyl factor $\Omega$ :

$$
\mathcal{I}^{\varepsilon}[\Omega, A]=-\frac{1}{4} \int d^{4-\varepsilon} x \sqrt{|\eta|} \eta^{\rho \alpha} \eta^{\sigma \beta} e^{-\varepsilon \Omega} M^{-\varepsilon}\left(\frac{1}{e^{2}}-\frac{b}{\varepsilon}\right) F_{\rho \sigma}^{a} F_{\alpha \beta}^{a} .
$$

This implies that the Weyl variation of the renormalized effective action for the background field is no longer zero and is given by

$$
\delta \mathcal{I}[\eta, \Omega, A]=-\frac{b}{4} \int d^{4} x \sqrt{|\eta|} F^{2} \delta \Omega,
$$

consistent with the results obtained using the proper time regularization.

\subsection{Quantum effective action for a self-interacting scalar field}

For a conformally coupled scalar field $\varphi$, one can similarly determine the one-loop effective action $\mathcal{I}[g, \varphi]$. Integrating the $\mathcal{B}$ anomaly of (2.58) gives

$$
\mathcal{I}_{\mathcal{B}}[\eta, \Omega, \bar{\varphi}]=\frac{b \lambda}{4 !} \int d^{4} x \bar{\varphi}^{4}(x) \Omega(x),
$$

with $b$ given by (2.59) and $\lambda$ being the renormalized quartic coupling defined at the scale $M$. The flat space action obtained from standard computations gives

$$
\mathcal{I}[\eta, \bar{\varphi}]=-\int d^{4} x\left[\frac{1}{2}|\partial \bar{\varphi}|^{2}+\frac{\lambda}{4 !} \bar{\varphi}^{2}(x)\left(1+\frac{b}{2} \log \left(\frac{-\partial^{2}}{M^{2}}\right)\right) \bar{\varphi}^{2}(x)\right] .
$$

Using (2.46), the full effective action is given by

$$
\mathcal{I}[g, \varphi]=-\int d^{4} x\left[\frac{1}{2}|\partial \bar{\varphi}|^{2}+\frac{\lambda}{4 !} \bar{\varphi}^{2}(x)\left(1+\frac{b}{2} \log \left(\frac{-\partial^{2}}{M^{2}}\right)-b \Omega(x)\right) \bar{\varphi}^{2}(x)\right] .
$$

As in the case of the Yang-Mills action, the part of the action (3.18) arising from the anomaly does not require any weak-field approximation and is exact. The flat space action (3.19) is valid only assuming rapidly varying field. It could be evaluated though in other regimes of interest using techniques such as the large proper time expansion developed in $[67,68]$ or the Coleman-Weinberg method. However, note that when the field $\varphi$ is in the Coleman-Weinberg regime, the field $\bar{\varphi}$ may not be unless the scale factor is also slowly varying.

We see that the net effect of the Weyl anomaly in the combined action is to change the renormalization scale to an effective local renormalization scale $M(x):=M e^{\Omega(x)}$ consistent with (2.36). One can explain the answer intuitively if the scale factor is varying slowly 
compared to the typical scale of field variations (for example in a particle physics experiment in an expanding universe). In this case, one can use local momentum expansion to write $-\partial^{2}=k^{2}$. In local experiments (3.20) can be interpreted as a flat space action with momentum-squared $k^{2}$ but with a position dependent cutoff $M(x)$. One can equivalently interpret $k^{2} / M^{2}(x)$ as $p^{2}(x) / M^{2}$ in terms of physical momentum-squared $p^{2}=e^{-2 \Omega(x)} k^{2}$ with a fixed RG scale $M$. This suggests that we can use the local renormalization group to define a position-dependent 'running' coupling

$$
\begin{aligned}
\lambda\left(p^{2}(x)\right) & =\frac{\lambda}{1-\frac{b}{2} \log \left(\frac{p^{2}(x)}{M^{2}}\right)} \\
& =\lambda\left[1+\frac{b}{2} \log \left(\frac{p^{2}(x)}{M^{2}}\right)+\frac{b^{2}}{4} \log ^{2}\left(\frac{p^{2}(x)}{M^{2}}\right)+\ldots\right] .
\end{aligned}
$$

Equation (3.21) re-sums the leading logarithms to all orders as with the usual flat spacetime renormalization group but now locally. The effective coupling decreases as the universe expands because the beta function is positive.

The resulting RG-improved action satisfies the local renormalization group equation in much the same way the RG-improved action in flat space satisfies the usual renormalization group equation. Consequently, the RG-improved answer becomes better and better at late times even though naive perturbation theory would break down, thus extending the range of applicability of the perturbative computations.

In more general situations with a rapidly varying scale factor, one cannot use the momentum basis as above but equation (3.20) is still valid. One might be tempted to interpret the full answer in terms of the logarithm of the covariant d'Alembertian in curved spacetime, $\log \left(-\nabla^{2} / M^{2}\right)$. However, the full covariantization is rather nontrivial and requires many more nonlocal covariant terms which combine into a Weyl-invariant piece $[8,36]$. We discuss this in detail in section 3.5.

\subsection{Equations of motion}

If we are interested in the equations of motion of the fields in a fixed background metric, then the metric does not need to be varied and can be assumed to be Weyl flat. The equations of motion for the background Yang-Mills field follow straightforwardly from the action $\mathcal{I}[g, A](3.12)$ and are given by

$$
\frac{\delta \mathcal{I}[\eta, A]}{\delta A_{\mu}}+\frac{\delta \mathcal{I}_{\mathcal{B}}[\eta, \Omega, A]}{\delta A_{\mu}}=0
$$

The first term gives the logarithmic modifications to the flat space equations of motion arising from integrating out massless charged particles. The second term gives rise to the anomalous coupling to the conformal factor of the metric which breaks the Weyl invariance. Similar considerations extend to the equations of motion for the Weyl-transformed scalar $\bar{\varphi}$.

These actions are thus adequate for studying the equations of motion for the fluctuations of the gauge field or a scalar field in an arbitrary Robertson-Walker background including the full anomalous dependence on the Weyl factor. This is the situation one encounters, for example, in studying the primordial perturbations of a scalar or of the electromagnetic field in a slowly rolling inflationary background. 


\subsection{Barvinsky-Vilkovisky expansion and conformal decomposition}

In the weak curvature limit, we can compare our results with the covariant curvature expansion developed by Barvinsky, Vilkovisky, and collaborators [3-8]. It provides a useful check on our results obtained using a rather different method which does not rely on the weak curvature approximation.

The main idea behind the Barvinsky-Vilkovisky (BV) expansion is to decompose the metric as $g_{\mu \nu}=\eta_{\mu \nu}+h_{\mu \nu}$ and treat the fluctuations $h_{\mu \nu}$ as perturbations. The heat equation satisfied by the kernel $K(s)$ can be solved perturbatively around flat space using the analog of the Dirac interaction picture in quantum mechanics. The perturbative answers are then 'covariantized' up to a given order to express them in terms of covariant derivatives and polynomials of generalized curvature tensors schematically denoted as $\mathcal{R}$, which includes both terms like $R_{\mu \nu}$ as well as $F_{\mu \nu}$. This expansion is valid for small generalized curvatures but for the entire range of the proper time $s$. The effective action can thus be obtained by evaluating the integral (2.17). The final answer can be expressed in terms of non-local 'form factors' and schematically takes the form

$\mathcal{S}=\mathcal{S}_{0}+\mathcal{S}_{1}^{l o c}+\int d^{d} x \sqrt{|g|} \sum_{i=1}^{5} f_{i}\left(-\nabla_{2}^{2}\right) \mathcal{R}_{1} \mathcal{R}_{2}+\sum_{i=1}^{29} \mathcal{F}_{i}\left(-\nabla_{1}^{2},-\nabla_{2}^{2},-\nabla_{3}^{2}\right) \mathcal{R}_{1} \mathcal{R}_{2} \mathcal{R}_{3}(i)+\mathcal{O}\left(\mathcal{R}^{4}\right)$.

The notation is a shorthand for terms containing all possible combinations of curvatures such as $R f_{R R}\left(-\nabla^{2}\right) R$ and $\mathcal{F}_{2}\left(-\nabla_{1}^{2},-\nabla_{2}^{2},-\nabla_{3}^{2}\right) F_{1}{ }_{\nu}{ }_{\nu} F_{2}{ }_{\sigma}{ }_{\sigma} R_{3}{ }^{\sigma}{ }_{\mu}$, for example. The form factors $f$ and $\mathcal{F}$ as functions of the covariant Laplacian are generically non-local operators, and are to be understood as properly convoluted with the functions they act upon [4]. A similar result has been obtained for massless quantum electrodynamics by a somewhat different method by Donoghue and El-Menoufi $[35,36]$ by evaluating the one-loop Feynman diagrams for small metric fluctuations around flat space and then covariantizing the answers.

An important advantage of this expansion is that it gives all nonlocal terms in the action directly to a given order in perturbation theory. The price to pay though is that these expressions are necessarily perturbative, valid only in the regime of $\mathcal{R}^{2} \ll \nabla^{2} \mathcal{R}$. Note that there are two perturbative expansions at work. The loop expansion parameter is $e^{2}$ or $\lambda$, while the $\mathrm{BV}$ expansion involves a further approximation which treats the field perturbations, such as $h_{\mu \nu}, A_{\mu}$ or $V^{\prime \prime}$ as small. This weak field approximation implies that terms of the form $\partial^{2} h \partial^{2} h$ are to be regarded as much smaller than terms of the form $\partial^{4} h$ even though both have the same number of derivatives. Upon covariantization, it implies that the BV expansion is valid if $R^{2} \ll \nabla^{2} R$. By contrast, the local Schwinger-DeWitt expansion is valid for short proper time $\epsilon R \ll 1$ or equivalently for the entire weak gravity regime $R \ll M_{0}^{2}$ without any further restrictions on curvatures.

To compare (3.24) with our results, it is necessary to go to third order in the BV expansion. Explicit expressions to this order have been worked out in [6] but they are rather complicated going over several pages. It is not immediately obvious how these expressions could reduce to the simple expressions that we obtained earlier. However, one can use the fact that the Weyl variation of the BV effective action must correctly 
reproduce the local Weyl anomaly. This observation suggests a 'conformal decomposition' of the action in terms of a Weyl-invariant piece and a Weyl-variant piece [7, 8, 36, 69]. This conformal decomposition is what is most easily compared with our results.

To illustrate the idea, consider the BV effective action for quantum electrodynamics obtained by integrating out massless charged fields in the presence a background gauge field $A$. To third order in curvatures it is given by [36]:

$$
\begin{aligned}
\mathcal{I}[g, A]= & -\frac{1}{4} \int d^{4} x \sqrt{|g|}\left\{\frac{1}{e^{2}} F_{\mu \nu} F^{\mu \nu}-\frac{b}{2}\left[F^{\mu \nu} \log \left(\frac{-\nabla^{2}}{M^{2}}\right) F_{\mu \nu}+\frac{1}{3} F^{2} \frac{1}{\nabla^{2}} R+\right.\right. \\
& +4 R^{\mu \nu} \frac{1}{\nabla^{2}}\left(\log \left(\frac{-\nabla^{2}}{M^{2}}\right)\left(F_{\mu \sigma}^{a} F_{\nu}^{a} \sigma-\frac{1}{4} g_{\mu \nu} F^{2}\right)-F_{\mu \sigma} \log \left(\frac{-\nabla^{2}}{M^{2}}\right) F_{\nu}^{\sigma}+\right. \\
& \left.\left.+\frac{1}{4} g_{\mu \nu} F^{\alpha \beta} \log \left(\frac{-\nabla^{2}}{M^{2}}\right) F_{\alpha \beta}\right)-\frac{1}{3} R F^{\mu \nu} \frac{1}{\nabla^{2}} F_{\mu \nu}+W_{\beta \mu \nu}^{\alpha} F_{\alpha}{ }^{\beta} \frac{1}{\nabla^{2}} F^{\mu \nu}\right]+ \\
& \left.+4 \tilde{b} F^{\mu \nu} F_{\alpha}{ }^{\beta} \frac{1}{\nabla^{2}} W_{\beta \mu \nu}^{\alpha}\right\}+\mathcal{O}\left(\mathcal{R}^{4}\right)
\end{aligned}
$$

where the logarithm of the covariant d'Alembertian $\log \left(-\nabla^{2} / M^{2}\right)$ is defined as in (3.10) and

$$
b=\frac{1}{24 \pi^{2}}\left(N_{S}+4 N_{F}\right), \quad \tilde{b}=\frac{1}{96 \pi^{2}}\left(-N_{S}+2 N_{F}\right) .
$$

Note that $b$ is the usual beta function coefficient (2.57) in flat space but $\tilde{b}$ is relevant only in curved backgrounds. We have ignored the purely gravitational terms coming from the $\mathcal{C}$ anomaly that are independent of the background gauge field.

It turns out that except for the second term in the square bracket, all other terms in (3.25) are actually Weyl invariant $[8,36]$. This 'conformal decomposition' then implies that the only Weyl-variant term that could contribute to the $\mathcal{B}$ anomaly is precisely this second term:

$$
\tilde{\mathcal{I}}_{\mathcal{B}}[g, A]=-\frac{b}{4} \int d^{4} x \sqrt{|g|} F^{\mu \nu}\left(-\frac{1}{6} \frac{1}{\nabla^{2}} R\right) F_{\mu \nu} .
$$

Since all other terms taken together are Weyl invariant, for a Weyl-flat metric they must reduce to the one-loop effective action on flat space (3.9):

$$
\mathcal{I}[\eta, A]=-\frac{1}{4} \int d^{4} x F^{\mu \nu}\left[\frac{1}{e^{2}(M)}-\frac{b}{2} \log \left(\frac{-\partial^{2}}{M^{2}}\right)\right] F_{\mu \nu} .
$$

Hence for a Weyl-flat metric the action (3.25) simplifies dramatically to

$$
\mathcal{I}[g, A]=\mathcal{I}[\eta, A]+\tilde{\mathcal{I}}_{\mathcal{B}}[\eta, \Omega, A] .
$$

We would like to compare this result with the one obtained by integrating the anomaly:

$$
\mathcal{I}[g, A]=\mathcal{I}[\eta, A]+\mathcal{I}_{\mathcal{B}}[\eta, \Omega, A], \quad \text { with } \quad \mathcal{I}_{\mathcal{B}}[\eta, \Omega, A]=-\frac{b}{4} \int d^{4} x \eta^{\mu \rho} \eta^{\nu \sigma} F_{\mu \nu} \Omega(x) F_{\rho \sigma} .
$$

To this end, we note that the Weyl factor $\Omega[g](x)$ can be expressed as a nonlocal covariant functional of the metric $[9,70,71]$ given by

$$
\Omega[g](x)=\frac{1}{4} \int d^{4} y \sqrt{|g|} G_{4}(x, y) F_{4}[g](y),
$$


where

$$
F_{4}[g]:=E_{4}[g]-\frac{2}{3} \nabla^{2} R[g]=\left(R_{\mu \nu \rho \sigma} R^{\mu \nu \rho \sigma}-4 R_{\mu \nu} R^{\mu \nu}+R^{2}-\frac{2}{3} \nabla^{2} R\right)[g],
$$

and the Green function $G_{4}(x, y)$ defined by

$$
\Delta_{4}^{x}[g] G_{4}(x, y)=\delta^{(4)}(x, y):=\frac{\delta^{(4)}(x-y)}{\sqrt{|g|}}
$$

is the inverse of the Weyl-covariant quartic differential operator

$$
\Delta_{4}[g]=\left(\nabla^{2}\right)^{2}+2 R^{\mu \nu} \nabla_{\mu} \nabla_{\nu}+\frac{1}{3}\left(\nabla^{\nu} R\right) \nabla_{\nu}-\frac{2}{3} R \nabla^{2} .
$$

The expression (3.31) follows from the fact that for metrics related by a Weyl rescaling $g_{\mu \nu}=e^{2 \Omega(x)} \eta_{\mu \nu}$, the corresponding $F_{4}$ scalars are related by

$$
F_{4}[g]=e^{-4 \Omega}\left(F_{4}[\eta]+4 \Delta_{4}[\eta] \Omega\right)
$$

and the operators $\Delta_{4}$ by

$$
\Delta_{4}[g]=e^{-4 \Omega} \Delta_{4}[\eta]
$$

Since the Minkowski reference metric satisfies $F_{4}[\eta]=0$ the expression (3.31) follows from inverting (3.35). This expression is manifestly covariant but nonlocal, consistent with the fact that the anomalous $\Omega$ dependence represents genuine long-distance quantum effects that cannot be removed by counter-terms that are local functionals of the metric.

When $R^{2} \ll \nabla^{2} R$ one can expand the expression for $\Omega$ (3.31) in curvatures to obtain

$$
\Omega[g](x)=-\frac{1}{6} \frac{1}{\nabla^{2}} R+\mathcal{O}\left(R^{2}\right)
$$

which when substituted in (3.30) reproduces the anomaly action obtained in the BV regime (3.27).

To recover the full expression for $\Omega$ in the Barvinsky-Vilkovisky formalism one must invert the operator $\Delta_{4}$ perturbatively, which involves higher and higher orders in curvatures. As a result, the expression (3.31) for $\Omega$ will similarly involve terms to arbitrary order in the curvature expansion. This implies that to recover the exact and simple expression (3.12) obtained by integrating the Weyl anomaly it would be necessary to re-sum the covariant perturbation theory (3.25) to all orders in curvatures $R$ for the class of Weyl-flat metrics. Since $\mathcal{I}_{\mathcal{B}}$ already contains $F^{2}$, the next correction is of order $F^{2} R^{2} \sim \mathcal{R}^{4}$ in the generalized curvature expansion. In other words,

$$
\mathcal{I}_{\mathcal{B}}[\eta, \Omega, A]=\tilde{\mathcal{I}}_{\mathcal{B}}[\eta, \Omega, A]+\mathcal{O}\left(\mathcal{R}^{4}\right)
$$

Already at order $\mathcal{R}^{4}$, the expression in the BV expansion becomes unmanageable. It is remarkable that the simple expression (2.46) re-sums this expansion to all orders albeit for a restricted class of Weyl-flat metrics.

Thus, explicit 'covariantization' of our answer obtained by integrating the anomaly can lead to rather complicated expressions even though the exact answer (3.30) is strikingly 
simple. As noted earlier, our procedure guarantees that the full answer depends only the physical metric $g$ even though a priori the right hand side appears to depend on $\eta_{\mu \nu}$ and $\Omega$ separately.

Note that our action is valid on a special submanifold of Weyl-flat metrics in the field space of metrics. There is no way to work back from our action (3.30) valid for Weyl-flat metrics to obtain the full action (3.25) valid for a general metric. Information from the anomaly is not sufficient for this purpose, unlike in two dimensions for the Polyakov action.

Finally, by imposing different gauges one gets different nonlocal covariant expressions for $\Omega(x) .{ }^{11}$ The curvature expansion of these different expressions is not expected to be the same. This would hence require, for each gauge, a different identification of the Weylinvariant terms and the Weyl-anomalous ones in the curvature expansion. This is consistent with the fact that there are different conformal decompositions of the covariant effective action [8]. However, for any Weyl-flat metric the Weyl invariant terms must reduce to the flat space action, and the compact expression for the anomalous effective action is exact and unique in terms of $\Omega(x)$ and $\eta$.

\section{Acknowledgments}

A major part of this work was conducted within the framework of the ILP LABEX (ANR10-LABX-63) supported by French state funds managed by the Agence National de la Recherche within the Investissements d'Avenir programme under reference ANR-11-IDEX0004-02, and by the project QHNS in the program ANR Blanc SIMI5. T.B. and A.D. acknowledge the support from the Indo-French Centre CEFIPRA, under project number 5204-4. T. B. especially thanks the ICTP and the three host Indian institutes under the CEFIPRA project (the Department of Theoretical Physics at the Tata Institute of Fundamental Research, the International Centre for Theoretical Sciences, and the Harish-Chandra Research Institute) for the hospitality and stimulating environment provided during part of this work. We thank Hadi Godazgar, Alba Grassi, Takeshi Kobayashi, Roberto Percacci, Adam Schwimmer, Ashoke Sen, and Stefan Theisen for useful discussions.

Open Access. This article is distributed under the terms of the Creative Commons Attribution License (CC-BY 4.0), which permits any use, distribution and reproduction in any medium, provided the original author(s) and source are credited.

\section{References}

[1] J.S. Schwinger, The Theory of quantized fields. 1., Phys. Rev. 82 (1951) 914 [InSPIRE].

[2] B.S. DeWitt, Dynamical theory of groups and fields, North Carolina University, Chapel Hill U.S.A. (1963).

\footnotetext{
${ }^{11}$ For instance, since the Minkowski metric is Ricci flat $R[\eta]=0$, one can impose the R-flat gauge by inverting an analogous relation to $(3.35)$ but for the Ricci scalars $R[g]=e^{-2 \Omega}\left(R[\eta]-6 \nabla_{\eta}^{2} \Omega-6\left(\nabla_{\eta} \Omega\right)^{2}\right)$. The resulting expression for the conformal factor is $\Omega[g](x)=-\ln \left(1-\frac{1}{6} \int d y \bar{G}(x, y) R[g](y)\right)$ where $\bar{G}(x, y)$ is the Green function of the differential operator $-\nabla^{2}+\frac{1}{6} R[g]$.
} 
[3] A.O. Barvinsky and G.A. Vilkovisky, The generalized Schwinger-Dewitt technique and the unique effective action in quantum gravity, Phys. Lett. B 131 (1983) 313 [INSPIRE].

[4] A.O. Barvinsky and G.A. Vilkovisky, The Generalized Schwinger-Dewitt Technique in Gauge Theories and Quantum Gravity, Phys. Rept. 119 (1985) 1 [inSPIRE].

[5] A.O. Barvinsky and G.A. Vilkovisky, The Effective Action in Quantum Field Theory: Two Loop Approximation, Quantum Field Theory and Quantum Statistics 1 (1988) 245.

[6] A.O. Barvinsky, Yu. V. Gusev, G.A. Vilkovisky and V.V. Zhytnikov, The Basis of nonlocal curvature invariants in quantum gravity theory. (Third order.), J. Math. Phys. 35 (1994) 3525 [gr-qc/9404061] [InSPIRE].

[7] A.O. Barvinsky, Yu. V. Gusev, G.A. Vilkovisky and V.V. Zhytnikov, The One loop effective action and trace anomaly in four-dimensions, Nucl. Phys. B 439 (1995) 561 [hep-th/9404187] [INSPIRE].

[8] A.O. Barvinsky, A.G. Mirzabekian and V.V. Zhytnikov, Conformal decomposition of the effective action and covariant curvature expansion, in Proceedings of 6th Seminar on Quantum gravity, Moscow Russia (1995) [gr-qc/9510037] [INSPIRE].

[9] R.J. Riegert, A Nonlocal Action for the Trace Anomaly, Phys. Lett. 134B (1984) 56 [INSPIRE].

[10] S. Deser, Conformal anomalies: Recent progress, Helv. Phys. Acta 69 (1996) 570 [hep-th/9609138] [INSPIRE].

[11] J. Erdmenger and H. Osborn, Conserved currents and the energy momentum tensor in conformally invariant theories for general dimensions, Nucl. Phys. B 483 (1997) 431 [hep-th/9605009] [INSPIRE].

[12] J. Erdmenger, Conformally covariant differential operators: Properties and applications, Class. Quant. Grav. 14 (1997) 2061 [hep-th/9704108] [INSPIRE].

[13] S. Deser, Conformal anomalies revisited: Closed form effective actions in $D \geq 4$, Nucl. Phys. Proc. Suppl. 88 (2000) 204 [inSPIRE].

[14] A. Dabholkar, J. Gomes and S. Murthy, Localization \& Exact Holography, JHEP 04 (2013) 062 [arXiv:1111.1161] [INSPIRE].

[15] A. Dabholkar, J. Gomes and S. Murthy, Nonperturbative black hole entropy and Kloosterman sums, JHEP 03 (2015) 074 [arXiv: 1404.0033] [INSPIRE].

[16] A. Sen, Logarithmic Corrections to $N=2$ Black Hole Entropy: An Infrared Window into the Microstates, Gen. Rel. Grav. 44 (2012) 1207 [arXiv:1108.3842] [INSPIRE].

[17] S. Banerjee, R.K. Gupta, I. Mandal and A. Sen, Logarithmic Corrections to $N=4$ and $N=8$ Black Hole Entropy: A One Loop Test of Quantum Gravity, JHEP 11 (2011) 143 [arXiv:1106.0080] [INSPIRE].

[18] M.S. Turner and L.M. Widrow, Inflation Produced, Large Scale Magnetic Fields, Phys. Rev. D 37 (1988) 2743 [inSPIRE].

[19] B. Ratra, Cosmological 'seed' magnetic field from inflation, Astrophys. J. 391 (1992) L1 [INSPIRE].

[20] V. Demozzi, V. Mukhanov and H. Rubinstein, Magnetic fields from inflation?, JCAP 08 (2009) 025 [arXiv: 0907.1030] [INSPIRE]. 
[21] B.K. El-Menoufi, Inflationary magnetogenesis and non-local actions: The conformal anomaly, JCAP 02 (2016) 055 [arXiv:1511.02876] [INSPIRE].

[22] A. Benevides, A. Dabholkar and T. Kobayashi, Weyl Anomalies and Primordial Magnetogenesis, to appear (2018).

[23] A. Dolgov, Breaking of conformal invariance and electromagnetic field generation in the universe, Phys. Rev. D 48 (1993) 2499 [hep-ph/9301280] [InSPIRE].

[24] A. Dabholkar, Quantum Weyl Invariance and Cosmology, Phys. Lett. B 760 (2016) 31 [arXiv: 1511.05342] [INSPIRE].

[25] T. Bautista and A. Dabholkar, Quantum Cosmology Near Two Dimensions, Phys. Rev. D 94 (2016) 044017 [arXiv: 1511.07450] [INSPIRE].

[26] T. Bautista, A. Benevides, A. Dabholkar and A. Goel, Quantum Cosmology in Four Dimensions, arXiv:1512.03275 [INSPIRE].

[27] E. Mottola and R. Vaulin, Macroscopic Effects of the Quantum Trace Anomaly, Phys. Rev. D 74 (2006) 064004 [gr-qc/0604051] [INSPIRE].

[28] S. Deser and R.P. Woodard, Nonlocal Cosmology, Phys. Rev. Lett. 99 (2007) 111301 [arXiv:0706.2151] [INSPIRE].

[29] S. Nojiri and S.D. Odintsov, Modified non-local- $F(R)$ gravity as the key for the inflation and dark energy, Phys. Lett. B 659 (2008) 821 [arXiv:0708.0924] [INSPIRE].

[30] S. Jhingan, S. Nojiri, S.D. Odintsov, M. Sami, I. Thongkool and S. Zerbini, Phantom and non-phantom dark energy: The Cosmological relevance of non-locally corrected gravity, Phys. Lett. B 663 (2008) 424 [arXiv:0803.2613] [INSPIRE].

[31] S. Park and S. Dodelson, Structure formation in a nonlocally modified gravity model, Phys. Rev. D 87 (2013) 024003 [arXiv: 1209.0836] [InSPIRE].

[32] R.P. Woodard, Nonlocal Models of Cosmic Acceleration, Found. Phys. 44 (2014) 213 [arXiv: 1401.0254] [INSPIRE].

[33] J.F. Donoghue and B.K. El-Menoufi, Nonlocal quantum effects in cosmology: Quantum memory, nonlocal FLRW equations and singularity avoidance, Phys. Rev. D 89 (2014) 104062 [arXiv:1402.3252] [INSPIRE].

[34] H. Godazgar, K.A. Meissner and H. Nicolai, Conformal anomalies and the Einstein Field Equations, JHEP 04 (2017) 165 [arXiv: 1612.01296] [INSPIRE].

[35] J.F. Donoghue and B.K. El-Menoufi, QED trace anomaly, non-local Lagrangians and quantum Equivalence Principle violations, JHEP 05 (2015) 118 [arXiv:1503.06099] [INSPIRE].

[36] J.F. Donoghue and B.K. El-Menoufi, Covariant non-local action for massless QED and the curvature expansion, JHEP 10 (2015) 044 [arXiv: 1507.06321] [INSPIRE].

[37] A. Benevides and A. Dabholkar, Weyl Anomalies and Quantum Effective Actions in Cosmological Spacetimes, to appear (2017).

[38] L.F. Abbott, Introduction to the Background Field Method, Acta Phys. Polon. B 13 (1982) 33 [INSPIRE].

[39] R. Percacci, 100 Years of General Relativity. Vol. 3: An Introduction to Covariant Quantum Gravity and Asymptotic Safety, World Scientific, Singapore (2017). 
[40] D.V. Vassilevich, Heat kernel expansion: User's manual, Phys. Rept. 388 (2003) 279 [hep-th/0306138] [INSPIRE].

[41] S. Deser and A. Schwimmer, Geometric classification of conformal anomalies in arbitrary dimensions, Phys. Lett. B 309 (1993) 279 [hep-th/9302047] [INSPIRE].

[42] A. Codello, G. D'Odorico, C. Pagani and R. Percacci, The Renormalization Group and Weyl-invariance, Class. Quant. Grav. 30 (2013) 115015 [arXiv:1210.3284] [InSPIRE].

[43] D.M. Capper and M.J. Duff, The one loop neutrino contribution to the graviton propagator, Nucl. Phys. B 82 (1974) 147 [inSPIRE].

[44] S. Deser, M.J. Duff and C.J. Isham, Nonlocal Conformal Anomalies, Nucl. Phys. B 111 (1976) 45 [inSPIRE].

[45] M.J. Duff, Twenty years of the Weyl anomaly, Class. Quant. Grav. 11 (1994) 1387 [hep-th/9308075] [INSPIRE].

[46] I.T. Drummond and G.M. Shore, Conformal Anomalies for Interacting Scalar Fields in Curved Space-Time, Phys. Rev. D 19 (1979) 1134 [InSPIRE].

[47] H. Osborn, Weyl consistency conditions and a local renormalization group equation for general renormalizable field theories, Nucl. Phys. B 363 (1991) 486 [INSPIRE].

[48] I. Jack and H. Osborn, Analogs for the c Theorem for Four-dimensional Renormalizable Field Theories, Nucl. Phys. B 343 (1990) 647 [InSPIRE].

[49] G.M. Shore, A Local Renormalization Group Equation, Diffeomorphisms and Conformal Invariance in $\sigma$ Models, Nucl. Phys. B 286 (1987) 349 [InSPIRE].

[50] G.M. Shore, New methods for the renormalization of composite operator Green functions, Nucl. Phys. B 362 (1991) 85 [INSPIRE].

[51] H. Osborn, Renormalization and Composite Operators in Nonlinear $\sigma$ Models, Nucl. Phys. B 294 (1987) 595 [InSPIRE].

[52] H. Osborn, Derivation of a Four-dimensional c Theorem, Phys. Lett. B 222 (1989) 97 [INSPIRE].

[53] P.B. Gilkey, Invariance theory, the heat equation, and the Atiyah-Singer index theorem, CRC Press, Boca Raton U.S.A.(1984).

[54] R.T. Seeley, Complex powers of an elliptic operator, Proc. Symp. Pure Math. 10 (1967) 288.

[55] R. Seeley, The resolvent of an elliptic boundary problem, Am. J. Math. 91 (1969) 889.

[56] J. Hadamard, Lectures on Cauchy's problem in linear partial differential equations, Courier Corporation, Chelmsford U.S.A. (2014).

[57] S. Minakshisundaram and A. Pleijel, Some properties of the eigenfunctions of the Laplace operator on Riemannian manifolds, Can. J. Math. 1 (1949) 242 [INSPIRE].

[58] S. Minakshisundaram, Eigenfunctions on Riemannian manifolds, J. Indian Math. Soc. 17 (1953) 158.

[59] B.S. DeWitt, Dynamical theory of groups and fields, Conf. Proc. C 630701 (1964) 585 [INSPIRE].

[60] B.S. DeWitt, Quantum Theory of Gravity. 1. The Canonical Theory, Phys. Rev. 160 (1967) 1113 [INSPIRE]. 
[61] B.S. DeWitt, Quantum Theory of Gravity. 2. The Manifestly Covariant Theory, Phys. Rev. 162 (1967) 1195 [INSPIRE].

[62] B.S. DeWitt, Quantum Theory of Gravity. 3. Applications of the Covariant Theory, Phys. Rev. 162 (1967) 1239 [inSPIRE].

[63] G. Gibbons, in General Relativity: An Einstein Centenary Survey, S.W. Hawking and W. Israel eds., Cambridge University Press, Cambridge U.K. (1979).

[64] A. Barvinsky, Heat kernel expansion in the background field formalism, Scholarpedia 10 (2015) 31644.

[65] L. Bonora, P. Cotta-Ramusino and C. Reina, Conformal Anomaly and Cohomology, Phys. Lett. 126B (1983) 305 [INSPIRE].

[66] P.O. Mazur and E. Mottola, Weyl cohomology and the effective action for conformal anomalies, Phys. Rev. D 64 (2001) 104022 [hep-th/0106151] [INSPIRE].

[67] A.O. Barvinsky and V.F. Mukhanov, New nonlocal effective action, Phys. Rev. D 66 (2002) 065007 [hep-th/0203132] [InSPIRE].

[68] A.O. Barvinsky, Yu. V. Gusev, V.F. Mukhanov and D.V. Nesterov, Nonperturbative late time asymptotics for heat kernel in gravity theory, Phys. Rev. D 68 (2003) 105003 [hep-th/0306052] [INSPIRE].

[69] A.G. Mirzabekian, G.A. Vilkovisky and V.V. Zhytnikov, Partial summation of the nonlocal expansion for the gravitational effective action in four-dimensions, Phys. Lett. B 369 (1996) 215 [hep-th/9510205] [INSPIRE].

[70] E.S. Fradkin and G.A. Vilkovisky, Conformal Invariance and Asymptotic Freedom in Quantum Gravity, Phys. Lett. B 77 (1978) 262 [InSPIRE].

[71] S. Paneitz, A Quartic Conformally Covariant Differential Operator for Arbitrary Pseudo-Riemannian Manifolds (Summary), SIGMA 4 (2008) 036 [arXiv:0803.4331]. 